\title{
Impact of Lockdown on the Attitude of University Students in South India-A Cross-Sectional Observational Study
}

\author{
Reena Rachel John ${ }^{1}$ (D) Rohit P. John ${ }^{2}$
}

Received: 24 November 2020/ Accepted: 19 December 2020

(C) The Association of Oral and Maxillofacial Surgeons of India 2021

\begin{abstract}
Introduction The whole world is under the effects of the COVID-19 pandemic which has put all nations in a unique lockdown situation to contain the disease. Though the environment got ameliorated with low pollution levels, this unexpected lockdown has taken a toll on humans' mental health. As the effects of lockdown can affect college students due to fear, anxiety and stress, this study intends to assess the attitude of the university students.

Methodology An online questionnaire survey comprising 15 questions was conducted among four South Indian state university students. Their responses were recorded through Google Forms. The submitted responses were analysed using SPSS software version 17.

Results In total, 1241 responses were recorded from university students belonging to various faculties. Though $43 \%$ considered this lockdown as a convenient break, $60 \%$ could not focus on their studies. The importance of renewing relationships was felt by $47 \%$, and $83 \%$ tried to learn a new skill. Students from Tamil Nadu were looking forward towards a brighter future after the lockdown.

Conclusion This survey conducted in the early days of lockdown indicated that the university students were not anxious about the long-term effects of the pandemic but showed a decline in their academic performance.
\end{abstract}

Reena Rachel John

drreenaracheljohn@vmsdc.edu.in

1 Department of Oral and Maxillofacial Surgery, Vinayaka Mission's Sankarachariyar Dental College, Vinayaka Mission's Research Foundation-Deemed To Be University, Ariyanor, Salem, India

2 SDM College of Dental Sciences, Dharwad, Hubli, India
Keywords COVID-19 - Lockdown · University students · Coping $\cdot$ Attitude

\section{Introduction}

A viral outbreak is believed to have begun in Wuhan City of central Hubei Province of China in December 2019 [1]. It was established by WHO and the Chinese authorities that the etiological agent for this virus outbreak was the new virus named novel coronavirus (2019-nCov) [2]. The infection spread worldwide over a few weeks at a rapid pace. By 11 March 2020, the new coronavirus disease was declared a pandemic; as by then, nearly 114 countries were affected (WHO 2020) [3].

In order to contain the infection, a state of lockdown was imposed on many parts of the world. The economic setback and the increasing morbidity and mortality have been devastating globally. The mortality rates are steadily increasing, and the total number of deaths due to this virus has exceeded those caused by its predecessor-SARS [4].

It is an anticipated fact that this new pandemic COVID19 which has caused such destructive effects globally will result in confusion, anxiety and fear among the general public. As the understanding of this disease is evolving, the use of masks, sanitizers, physical distancing, self-isolation and quarantine has become the new normal in the present day scenario. This new normal would definitely add anguish among the individuals. The anxiety and concerns in society are affecting individuals to varying extent globally.

As per Ministry of Statistics and Programme Implementation, Government of India, the young generation forms $34.33 \%$ of the total population [5]. The mental health of the youth of the country is expected to be affected by the 
Table 1 State-wise comparison

\begin{tabular}{|c|c|c|c|c|c|c|c|c|}
\hline \multirow[t]{2}{*}{ No. } & \multirow[t]{2}{*}{ Item } & \multirow[t]{2}{*}{ Choices } & \multicolumn{4}{|c|}{ State-wise response (numbers in percentage) } & \multirow[t]{2}{*}{ Total } & \multirow[t]{2}{*}{$p$ value } \\
\hline & & & $\begin{array}{l}\text { Andhra } \\
\text { Pradesh }\end{array}$ & Karnataka & Kerala & $\begin{array}{l}\text { Tamil } \\
\text { Nadu }\end{array}$ & & \\
\hline \multirow[t]{3}{*}{1} & \multirow[t]{3}{*}{ I consider this lockdown } & $\begin{array}{l}\text { Convenient } \\
\text { break }\end{array}$ & $19(25 \%)$ & $\begin{array}{l}125 \\
(40.7 \%)\end{array}$ & $87(50 \%)$ & $\begin{array}{l}299 \\
(43.7 \%)\end{array}$ & $\begin{array}{l}530 \\
(42.7)\end{array}$ & 0.015 \\
\hline & & Inconvenience & $\begin{array}{l}31 \\
\quad(40.8 \%)\end{array}$ & $\begin{array}{l}107 \\
(34.9 \%)\end{array}$ & $47(27 \%)$ & $\begin{array}{l}206 \\
\quad(30.10 \%)\end{array}$ & $\begin{array}{l}391 \\
\quad(31.5 \%)\end{array}$ & \\
\hline & & Not sure & $\begin{array}{l}26 \\
(34.2 \%)\end{array}$ & $\begin{array}{l}75 \\
(24.4 \%)\end{array}$ & $40(23 \%)$ & $\begin{array}{l}179 \\
(26.2 \%)\end{array}$ & $\begin{array}{l}320 \\
(25.8 \%)\end{array}$ & \\
\hline \multirow[t]{3}{*}{2} & \multirow[t]{3}{*}{ Number of hours I sleep } & Increased & $57(75 \%)$ & $\begin{array}{l}217 \\
\quad(70.7 \%)\end{array}$ & $\begin{array}{l}143 \\
(82.2 \%)\end{array}$ & $\begin{array}{l}425 \\
\quad(62.10 \%)\end{array}$ & $\begin{array}{l}842 \\
\quad(67.8 \%)\end{array}$ & $<0.001$ \\
\hline & & Decreased & $2(2.6 \%)$ & $23(7.5 \%)$ & $6(3.4 \%)$ & $59(8.6 \%)$ & $90(7.3 \%)$ & \\
\hline & & No change & $\begin{array}{l}17 \\
\quad(22.4 \%)\end{array}$ & $\begin{array}{l}67 \\
(21.8 \%)\end{array}$ & $\begin{array}{l}25 \\
(14.4 \%)\end{array}$ & $\begin{array}{l}200 \\
(29.2 \%)\end{array}$ & $\begin{array}{l}309 \\
(24.9 \%)\end{array}$ & \\
\hline \multirow[t]{3}{*}{3} & \multirow[t]{3}{*}{ Screen viewing time } & Increased & $57(75 \%)$ & $\begin{array}{l}266 \\
(86.6 \%)\end{array}$ & $\begin{array}{l}147 \\
(84.5 \%)\end{array}$ & $\begin{array}{l}498 \\
\quad(72.8 \%)\end{array}$ & $\begin{array}{l}968 \\
(78 \%)\end{array}$ & $<0.001$ \\
\hline & & Decreased & $\begin{array}{l}11 \\
(14.5 \%)\end{array}$ & $23(7.5 \%)$ & $11(6.3 \%)$ & $68(9.9 \%)$ & $\begin{array}{l}113 \\
(9.1 \%)\end{array}$ & \\
\hline & & No change & $8(10.5 \%)$ & $18(5.9 \%)$ & $16(9.2 \%)$ & $\begin{array}{l}118 \\
(17.3 \%)\end{array}$ & $\begin{array}{l}160 \\
(12.9 \%)\end{array}$ & \\
\hline \multirow[t]{3}{*}{4} & \multirow[t]{3}{*}{ I am able to focus on my studies } & $\begin{array}{l}\text { More than } \\
\text { normal }\end{array}$ & $9(11.8 \%)$ & $\begin{array}{l}45 \\
(14.7 \%)\end{array}$ & $\begin{array}{l}26 \\
(14.9 \%)\end{array}$ & $\begin{array}{l}182 \\
(26.6 \%)\end{array}$ & $\begin{array}{l}262 \\
\quad(21.1 \%)\end{array}$ & $<0.001$ \\
\hline & & $\begin{array}{c}\text { Less than } \\
\text { normal }\end{array}$ & $\begin{array}{l}58 \\
\quad(76.3 \%)\end{array}$ & $\begin{array}{l}219 \\
\quad(71.3 \%)\end{array}$ & $\begin{array}{l}117 \\
(67.2 \%)\end{array}$ & $\begin{array}{l}348 \\
\quad(50.9 \%)\end{array}$ & $\begin{array}{l}742 \\
\quad(59.8 \%)\end{array}$ & \\
\hline & & No change & $9(11.8 \%)$ & $43(14 \%)$ & $\begin{array}{l}31 \\
\quad(17.8 \%)\end{array}$ & $\begin{array}{l}154 \\
(22.5 \%)\end{array}$ & $\begin{array}{l}237 \\
\quad(19.1 \%)\end{array}$ & \\
\hline \multirow[t]{3}{*}{5} & \multirow[t]{3}{*}{ This time enabled me to } & Relax & $\begin{array}{l}42 \\
\quad(55.3 \%)\end{array}$ & $\begin{array}{l}138 \\
(45 \%)\end{array}$ & $\begin{array}{l}93 \\
\quad(53.4 \%)\end{array}$ & $\begin{array}{l}210 \\
(30.7 \%)\end{array}$ & $\begin{array}{l}483 \\
\quad(38.9 \%)\end{array}$ & $<0.001$ \\
\hline & & Refocus & $7(9.2 \%)$ & $21(6.8 \%)$ & $13(7.5 \%)$ & $47(6.9 \%)$ & $88(7.1 \%)$ & \\
\hline & & Both & $\begin{array}{l}27 \\
\quad(35.5 \%)\end{array}$ & $\begin{array}{l}148 \\
\quad(48.2 \%)\end{array}$ & $\begin{array}{l}68 \\
(39.1 \%)\end{array}$ & $\begin{array}{l}427 \\
\quad(62.4 \%)\end{array}$ & $\begin{array}{l}670 \\
(54 \%)\end{array}$ & \\
\hline \multirow[t]{3}{*}{6} & \multirow[t]{3}{*}{ Academically I am/ feel } & $\begin{array}{l}\text { More prepared } \\
\text { and confident }\end{array}$ & $\begin{array}{l}11 \\
(14.5 \%)\end{array}$ & $\begin{array}{l}42 \\
\quad(13.7 \%)\end{array}$ & $\begin{array}{l}21 \\
\quad(12.1 \%)\end{array}$ & $\begin{array}{l}151 \\
\quad(22.1 \%)\end{array}$ & $\begin{array}{l}225 \\
\quad(18.1 \%)\end{array}$ & $<0.001$ \\
\hline & & $\begin{array}{l}\text { Less prepared } \\
\text { and confident }\end{array}$ & $\begin{array}{l}52 \\
\quad(68.4 \%)\end{array}$ & $\begin{array}{l}187 \\
(60.9 \%)\end{array}$ & $\begin{array}{l}109 \\
(62.6 \%)\end{array}$ & $\begin{array}{l}318 \\
\quad(46.5 \%)\end{array}$ & $\begin{array}{l}666 \\
(53.7 \%)\end{array}$ & \\
\hline & & No change & $\begin{array}{l}13 \\
(17.1 \%)\end{array}$ & $\begin{array}{l}78 \\
(25.4 \%)\end{array}$ & $\begin{array}{l}44 \\
(25.3 \%)\end{array}$ & $\begin{array}{l}215 \\
(31.4 \%)\end{array}$ & $\begin{array}{l}350 \\
\quad(28.2 \%)\end{array}$ & \\
\hline \multirow[t]{4}{*}{7} & \multirow[t]{4}{*}{ Lockdown has given me opportunity } & $\begin{array}{l}\text { Spend more } \\
\text { time with } \\
\text { loved ones }\end{array}$ & $\begin{array}{l}36 \\
(47.4 \%)\end{array}$ & $\begin{array}{l}159 \\
(51.8 \%)\end{array}$ & $\begin{array}{l}119 \\
\quad(68.4 \%)\end{array}$ & $\begin{array}{l}278 \\
(40.6 \%)\end{array}$ & $\begin{array}{l}592 \\
\quad(47.7 \%)\end{array}$ & $<0.001$ \\
\hline & & $\begin{array}{l}\text { Binge watch } \\
\text { shows }\end{array}$ & $8(10.5 \%)$ & $\begin{array}{l}42 \\
\quad(13.7 \%)\end{array}$ & $\begin{array}{l}23 \\
\quad(13.2 \%)\end{array}$ & $62(9.1 \%)$ & $\begin{array}{l}135 \\
(10.9 \%)\end{array}$ & \\
\hline & & $\begin{array}{l}\text { Spend more } \\
\text { time with } \\
\text { myself }\end{array}$ & $\begin{array}{l}21 \\
\quad(27.6 \%)\end{array}$ & $\begin{array}{l}78 \\
(25.4 \%)\end{array}$ & $\begin{array}{l}22 \\
(12.6 \%)\end{array}$ & $\begin{array}{l}228 \\
\quad(33.3 \%)\end{array}$ & $\begin{array}{l}349 \\
\quad(28.1 \%)\end{array}$ & \\
\hline & & $\begin{array}{l}\text { None of the } \\
\text { above }\end{array}$ & $\begin{array}{l}11 \\
(14.5 \%)\end{array}$ & $28(9.1 \%)$ & $10(5.7 \%)$ & $116(17 \%)$ & $\begin{array}{l}165 \\
(13.3 \%)\end{array}$ & \\
\hline \multirow[t]{2}{*}{8} & \multirow[t]{2}{*}{ Lockdown made me learn something new } & No & $\begin{array}{l}16 \\
(21.1 \%)\end{array}$ & $\begin{array}{l}64 \\
\quad(20.8 \%)\end{array}$ & $\begin{array}{l}36 \\
(20.7 \%)\end{array}$ & $97(14.2 \%)$ & $\begin{array}{l}213 \\
\quad(17.2 \%)\end{array}$ & 0.023 \\
\hline & & Yes & $\begin{array}{l}60 \\
(78.9 \%)\end{array}$ & $\begin{array}{l}243 \\
\quad(79.2 \%)\end{array}$ & $\begin{array}{l}138 \\
(79.3 \%)\end{array}$ & $\begin{array}{l}587 \\
\quad(85.8 \%)\end{array}$ & $\begin{array}{l}1028 \\
(82.8 \%)\end{array}$ & \\
\hline
\end{tabular}


Table 1 continued

\begin{tabular}{|c|c|c|c|c|c|c|c|c|}
\hline \multirow[t]{2}{*}{ No. } & \multirow[t]{2}{*}{ Item } & \multirow[t]{2}{*}{ Choices } & \multicolumn{4}{|c|}{ State-wise response (numbers in percentage) } & \multirow[t]{2}{*}{ Total } & \multirow[t]{2}{*}{$p$ value } \\
\hline & & & $\begin{array}{l}\text { Andhra } \\
\text { Pradesh }\end{array}$ & Karnataka & Kerala & $\begin{array}{l}\text { Tamil } \\
\text { Nadu }\end{array}$ & & \\
\hline \multirow[t]{2}{*}{9} & On a personal level I feel, this lockdown has done & $\begin{array}{l}\text { More harm } \\
\text { than good }\end{array}$ & $\begin{array}{l}29 \\
(38.2 \%)\end{array}$ & $\begin{array}{l}114 \\
\quad(37.1 \%)\end{array}$ & $\begin{array}{l}62 \\
(35.6 \%)\end{array}$ & $\begin{array}{l}229 \\
(33.5 \%)\end{array}$ & $\begin{array}{l}434 \\
(35 \%)\end{array}$ & 0.643 \\
\hline & & $\begin{array}{l}\text { More good } \\
\text { than harm }\end{array}$ & $\begin{array}{l}47 \\
\quad(61.8 \%)\end{array}$ & $\begin{array}{l}193 \\
\quad(62.9 \%)\end{array}$ & $\begin{array}{l}112 \\
\quad(64.4 \%)\end{array}$ & $\begin{array}{l}455 \\
\quad(66.5 \%)\end{array}$ & $\begin{array}{l}807 \\
\quad(65 \%)\end{array}$ & \\
\hline \multirow[t]{4}{*}{10} & Considering everything that I am going on, I feel & Safe & $\begin{array}{l}24 \\
(31.6 \%)\end{array}$ & $\begin{array}{l}101 \\
\quad(32.9 \%)\end{array}$ & $\begin{array}{l}57 \\
\quad(32.8 \%)\end{array}$ & $\begin{array}{l}260 \\
(38 \%)\end{array}$ & $\begin{array}{l}442 \\
\quad(35.6 \%)\end{array}$ & $<0.001$ \\
\hline & & Anxious & 9 & $\begin{array}{l}58 \\
\quad(18.9 \%)\end{array}$ & $\begin{array}{l}55 \\
(31.6 \%)\end{array}$ & $\begin{array}{l}103 \\
(15.1 \%)\end{array}$ & $\begin{array}{l}225 \\
\quad(18.1 \%)\end{array}$ & \\
\hline & & Scared & $\begin{array}{l}29 \\
\quad(38.2 \%)\end{array}$ & $40(13 \%)$ & $\begin{array}{l}22 \\
(12.6 \%)\end{array}$ & $\begin{array}{l}92 \\
\quad(13.5 \%)\end{array}$ & $\begin{array}{l}183 \\
\quad(14.7 \%)\end{array}$ & \\
\hline & & Uncertain & $\begin{array}{l}14 \\
(18.4 \%)\end{array}$ & $\begin{array}{l}108 \\
(35.2 \%)\end{array}$ & $40(23 \%)$ & $\begin{array}{l}229 \\
\quad(33.5 \%)\end{array}$ & $\begin{array}{l}391 \\
\quad(31.5 \%)\end{array}$ & \\
\hline \multirow[t]{4}{*}{11} & If I think of going back to normal life, I feel & Excited & $\begin{array}{l}14 \\
(18.4 \%)\end{array}$ & $\begin{array}{l}124 \\
\quad(40.4 \%)\end{array}$ & $\begin{array}{l}55 \\
(31.6 \%)\end{array}$ & $\begin{array}{l}282 \\
\quad(41.2 \%)\end{array}$ & $\begin{array}{l}475 \\
\quad(38.3 \%)\end{array}$ & $<0.001$ \\
\hline & & Panic & $\begin{array}{l}14 \\
(18.4 \%)\end{array}$ & $20(6.5 \%)$ & $\begin{array}{l}11 \\
(6.3 \%)\end{array}$ & $\begin{array}{l}76 \\
(11.1 \%)\end{array}$ & $\begin{array}{l}121 \\
\quad(9.8 \%)\end{array}$ & \\
\hline & & Tensed & $\begin{array}{l}31 \\
\quad(40.8 \%)\end{array}$ & $\begin{array}{l}108 \\
(35.2 \%)\end{array}$ & $\begin{array}{l}79 \\
(45.4 \%)\end{array}$ & $\begin{array}{l}177 \\
(25.9 \%)\end{array}$ & $\begin{array}{l}395 \\
\quad(31.8 \%)\end{array}$ & \\
\hline & & $\begin{array}{l}\text { Haven't } \\
\text { thought } \\
\text { about that }\end{array}$ & $\begin{array}{l}17 \\
(22.4 \%)\end{array}$ & $\begin{array}{l}55 \\
\quad(17.9 \%)\end{array}$ & $\begin{array}{l}29 \\
(16.7 \%)\end{array}$ & $\begin{array}{l}149 \\
(21.8 \%)\end{array}$ & $\begin{array}{l}250 \\
(20.1 \%)\end{array}$ & \\
\hline \multirow[t]{3}{*}{12} & $\begin{array}{l}\text { Looking back on times before lockdown I feel I } \\
\text { was }\end{array}$ & Overworked & $\begin{array}{l}28 \\
(36.8 \%)\end{array}$ & $\begin{array}{l}81 \\
(26.4 \%)\end{array}$ & ${ }^{45}(25.9 \%)$ & $\begin{array}{l}209 \\
(30.6 \%)\end{array}$ & $\begin{array}{l}363 \\
(29.3 \%)\end{array}$ & 0.332 \\
\hline & & $\begin{array}{l}\text { Enjoying } \\
\text { Routine }\end{array}$ & $34(44.7 \%)$ & $\begin{array}{l}142 \\
(46.3 \%)\end{array}$ & $87(50 \%)$ & $\begin{array}{l}298 \\
(43.6 \%)\end{array}$ & $\begin{array}{l}561 \\
(45.2 \%)\end{array}$ & \\
\hline & & $\begin{array}{l}\text { Not Doing } \\
\text { enough }\end{array}$ & $\begin{array}{l}14 \\
(18.4 \%)\end{array}$ & 84 (27.4\%) & $\begin{array}{l}42 \\
(24.1 \%)\end{array}$ & $\begin{array}{l}177 \\
(25.9 \%)\end{array}$ & $\begin{array}{l}317 \\
\quad(25.5 \%)\end{array}$ & \\
\hline \multirow[t]{2}{*}{13} & $\begin{array}{l}\text { On getting back to life after lockdown my } \\
\text { lifestyle/routine would }\end{array}$ & Change & $\begin{array}{l}48 \\
(63.2 \%)\end{array}$ & $\begin{array}{l}248 \\
\quad(80.8 \%)\end{array}$ & $\begin{array}{l}138 \\
(79.3 \%)\end{array}$ & $\begin{array}{l}537 \\
(78.5 \%)\end{array}$ & $\begin{array}{l}971 \\
\quad(78.2 \%)\end{array}$ & 0.009 \\
\hline & & Not change & 28 & $\begin{array}{l}59 \\
(19.2 \%)\end{array}$ & $\begin{array}{l}36 \\
(20.7 \%)\end{array}$ & $\begin{array}{l}147 \\
(21.5 \%)\end{array}$ & $\begin{array}{l}270 \\
(21.8 \%)\end{array}$ & \\
\hline 14 & $\begin{array}{l}\text { On a scale } 1-10 \text { how badly you want lockdown to } \\
\text { end }\end{array}$ & & 76 & 307 & 684 & 174 & 1241 & 0.16 \\
\hline \multirow[t]{3}{*}{15} & Future world according to me looks & Bright & 12 (15.8\%) & $\begin{array}{l}95 \\
\quad(30.9 \%)\end{array}$ & $\begin{array}{l}57 \\
\quad(32.8 \%)\end{array}$ & $\begin{array}{l}208 \\
\quad(30.4 \%)\end{array}$ & $\begin{array}{l}372 \\
\quad(30 \%)\end{array}$ & $<0.001$ \\
\hline & & Bleak & $\begin{array}{l}34 \\
\quad(44.7 \%)\end{array}$ & $\begin{array}{l}123 \\
(40.1 \%)\end{array}$ & $\begin{array}{l}88 \\
\quad(50.6 \%)\end{array}$ & $\begin{array}{l}262 \\
\quad(38.3 \%)\end{array}$ & $\begin{array}{l}507 \\
\quad(40.9 \%)\end{array}$ & \\
\hline & & $\begin{array}{c}\text { Same as } \\
\text { before }\end{array}$ & $\begin{array}{l}30 \\
\quad(39.5 \%)\end{array}$ & $89(29 \%)$ & $\begin{array}{l}29 \\
\quad(16.7 \%)\end{array}$ & $\begin{array}{l}214 \\
\quad(31.3 \%)\end{array}$ & $\begin{array}{l}362 \\
\quad(29.2 \%)\end{array}$ & \\
\hline
\end{tabular}

stress and anxiety created by the pandemic and the consequent isolation and quarantine experience. There is a paucity of research that evaluated the mental health concerns of the youth during this pandemic. Hence, this study was done through a questionnaire survey among the university students of the four South Indian states to understand the attitude and psychological impact of the pandemic on the young minds.

\section{Methodology}

This was a cross-sectional observational study carried out in South India. A snowball sampling technique was used. An online semi-structured questionnaire with closed-ended questions was developed using Google Forms with a consent form appended to it. The questionnaire was pretested among a small group of students within an institution and also validated by three professionals of Professor cadre in 
order to ascertain the level of understanding of the questions by the students.

The Google Forms questionnaire survey consisted of 15 questions addressing the attitude of the students towards the lockdown due to the pandemic, which was circulated among the university students through various social media like WhatsApp, Facebook and email. The link was shared by the contacts to their friends and contacts who further shared with their friends and contacts, which resulted in a snowballing effect. The study proposal had been approved by the Institutional Ethics Committee of Vinayaka Mission's Sankarachariyar Dental College, Vinayaka Mission's Research Foundation (Deemed to be University).

The Google Forms questionnaire was circulated among the university students of the states of Tamil Nadu, Karnataka, Kerala and Andhra Pradesh. The questionnaire survey was accessible for response for a period of three weeks. The data collection was initiated on 25 April 2020 at 10.00a.m. and was closed on 16 May 2020 at 10.00 p.m. IST.

The submitted responses were tabulated, and statistical analysis was performed using SPSS software version 17. The frequency, percentage, mean, standard deviation and $\mathrm{p}$ value were derived from the data obtained through the questionnaire survey.

\section{Results}

The online questionnaire survey was distributed among university students. A total of 1241 responses were recorded from university students of the faculty of medicine, dentistry, engineering and other faculties like arts and science stream. Among the faculties of study who responded to the survey, $66 \%$ were from the faculty of dentistry followed by $21 \%$ of the faculty of medicine and $7 \%$ from other faculties and $6 \%$ from the faculty of engineering. Comparison of the state-wise responses is given in Table 1. Age-wise comparison is given in Table 2.

\section{Attitude of the University Students to the Lockdown}

Among the university students, $43 \%$ considered this lockdown as a convenient break from routine, despite the fact that the survey was circulated after almost 30 days of the lockdown experience. Duration of sleep had increased in $68 \%$ of the university students. Screen viewing time had increased among $78 \%$ of the students. Age-wise comparison between individuals less than and more than 20 years also revealed similar results. Between the four states, students from Kerala (82\%) had the highest response of increased sleep hours $(p<0.001)$. Students from
Karnataka $(87 \%)$ had the highest response of increased screen viewing time $(p<0.001)$.

\section{Academic Activity during the Lockdown}

The focus on studies had reduced in $60 \%$ of students compared to pre-lockdown times. Fifty-four percentage of the students felt that the time that they got during the lockdown helped them to relax and refocus. Academically, $54 \%$ felt less confident and less prepared academically to face an examination. Between the junior and senior university students, $60 \%$ of the seniors felt that their focus on studies had decreased. The senior students $(62 \%)$ felt less confident in their academics $(p=0.02)$. Students from Andhra Pradesh (76\%) recorded the highest response of less than usual focus on studies, and $68 \%$ of these students were less prepared to face examinations $(p<0.001)$. Students from Tamil Nadu (62\%) recorded the highest number of students who used this time to relax and refocus $(p<0.001)$.

\section{Importance of Relationships}

Forty-seven percentage of the students felt that this lockdown had given them the opportunity to spend more time with their loved ones, while $28 \%$ felt that they were able to spend time with oneself. Among this group of students, $49 \%$ of the junior students spent more time with their loved ones $(p=0.015)$. It was observed that $68 \%$ of the students from Kerala spent more time with loved ones $(p<0.001)$.

\section{Manner of Coping with this Challenge}

With regards to facing this pandemic, $83 \%$ of the students tried to learn something new apart from their academic subjects. Anxiety questions directed at the students showed that $35 \%$ felt safe during this time, while the remaining felt anxious, scared and uncertain. Interestingly, the questions on outlook of the students on the future showed that $39 \%$ were excited about getting back to normal life and $20 \%$ had not even thought about the return back to normal life. Considering everything that is going on, the younger students $(41 \%)$ felt safe, while the older students $(33 \%)$ felt uncertain $(p<0.001)$.Considering all that is happening, students from Tamil Nadu $(38 \%)$ felt safe $(\mathrm{p}<0.001)$. Students from Tamil Nadu (41\%) and Karnataka (40\%) were excited about getting back to normal life $(p<0.001)$. Of the $41 \%$ of the total number of students who felt that the future looked bleak, $51 \%$ students were from Kerala $(p<0.001)$. Between states and age, the desire for the lockdown to end has not been statistically significant. $64.5 \%$ of the students felt that the lockdown period had done more good than harm. Overall perceptions between 
Table 2 Age-wise comparison

\begin{tabular}{|c|c|c|c|c|c|c|}
\hline \multirow[t]{2}{*}{ No. } & \multirow[t]{2}{*}{ Item } & \multirow[t]{2}{*}{ Choices } & \multicolumn{2}{|l|}{ Age } & \multirow[t]{2}{*}{ Total } & \multirow[t]{2}{*}{$p$ value } \\
\hline & & & $<20$ years & $>20$ years & & \\
\hline \multirow[t]{3}{*}{1} & \multirow[t]{3}{*}{ I consider this lockdown } & Convenient break & $\begin{array}{l}247 \\
(45.7 \%)\end{array}$ & $\begin{array}{l}315 \\
(41.5 \%)\end{array}$ & $\begin{array}{l}562 \\
\quad(43.2 \%)\end{array}$ & 0.052 \\
\hline & & Inconvenience & $\begin{array}{l}151 \\
(27.9 \%)\end{array}$ & $\begin{array}{l}260 \\
(34.3 \%)\end{array}$ & $\begin{array}{l}411 \\
(31.6 \%)\end{array}$ & \\
\hline & & Not sure & $\begin{array}{l}143 \\
(26.4 \%)\end{array}$ & $\begin{array}{l}184 \\
(24.2 \%)\end{array}$ & $\begin{array}{l}327 \\
\quad(25.2 \%)\end{array}$ & \\
\hline \multirow[t]{3}{*}{2} & \multirow[t]{3}{*}{ Number of hours I sleep } & Increased & $\begin{array}{l}361 \\
\quad(66.7 \%)\end{array}$ & $\begin{array}{l}523 \\
\quad(68.9 \%)\end{array}$ & $884(68 \%)$ & 0.458 \\
\hline & & Decreased & $38(7 \%)$ & $59(7.8 \%)$ & $97(7.5 \%)$ & \\
\hline & & No change & $\begin{array}{l}142 \\
(26.2 \%)\end{array}$ & $\begin{array}{l}177 \\
(23.3 \%)\end{array}$ & $\begin{array}{l}319 \\
\quad(24.5 \%)\end{array}$ & \\
\hline \multirow[t]{3}{*}{3} & \multirow[t]{3}{*}{ Screen viewing time } & Increased & $\begin{array}{l}410 \\
\quad(75.8 \%)\end{array}$ & $\begin{array}{l}604 \\
\quad(79.6 \%)\end{array}$ & $1014(78 \%)$ & 0.164 \\
\hline & & Decreased & $50(9.2 \%)$ & $68(9 \%)$ & $118(9.1 \%)$ & \\
\hline & & No change & $81(15 \%)$ & $87(11.5 \%)$ & $\begin{array}{l}168 \\
(12.9 \%)\end{array}$ & \\
\hline \multirow[t]{3}{*}{4} & \multirow[t]{3}{*}{ I am able to focus on my studies } & More than normal & $\begin{array}{l}110 \\
(20.3 \%)\end{array}$ & $\begin{array}{l}160 \\
(21.1 \%)\end{array}$ & $\begin{array}{l}270 \\
\quad(20.8 \%)\end{array}$ & 0.02 \\
\hline & & Less than normal & $\begin{array}{l}308 \\
\quad(56.9 \%)\end{array}$ & $\begin{array}{l}473 \\
\quad(62.3 \%)\end{array}$ & $\begin{array}{l}781 \\
\quad(60.1 \%)\end{array}$ & \\
\hline & & No change & $\begin{array}{l}123 \\
(22.7 \%)\end{array}$ & $\begin{array}{l}126 \\
(16.6 \%)\end{array}$ & $\begin{array}{l}249 \\
(19.2 \%)\end{array}$ & \\
\hline \multirow[t]{3}{*}{5} & \multirow[t]{3}{*}{ This time enabled me to } & Relax & $\begin{array}{l}204 \\
\quad(37.7 \%)\end{array}$ & $\begin{array}{l}305 \\
\quad(40.2 \%)\end{array}$ & $\begin{array}{l}509 \\
\quad(39.2 \%)\end{array}$ & 0.05 \\
\hline & & Refocus & $30(5.5 \%)$ & $64(8.4 \%)$ & $94(7.2 \%)$ & \\
\hline & & Both & $\begin{array}{l}307 \\
\quad(56.7 \%)\end{array}$ & $\begin{array}{l}390 \\
\quad(51.4 \%)\end{array}$ & $\begin{array}{l}697 \\
(53.6 \%)\end{array}$ & \\
\hline \multirow[t]{3}{*}{6} & \multirow[t]{3}{*}{ Academically I am/ feel } & $\begin{array}{l}\text { More prepared and } \\
\text { confident }\end{array}$ & $89(16.5 \%)$ & $\begin{array}{l}147 \\
(19.4 \%)\end{array}$ & $\begin{array}{l}236 \\
(18.2 \%)\end{array}$ & 0.38 \\
\hline & & Less prepared and confident & $\begin{array}{l}293 \\
(54.2 \%)\end{array}$ & $\begin{array}{l}403 \\
\quad(53.1 \%)\end{array}$ & $\begin{array}{l}696 \\
\quad(53.5 \%)\end{array}$ & \\
\hline & & No change & $\begin{array}{l}159 \\
(29.4 \%)\end{array}$ & $\begin{array}{l}209 \\
\quad(27.5 \%)\end{array}$ & $\begin{array}{l}368 \\
\quad(28.3 \%)\end{array}$ & \\
\hline \multirow[t]{4}{*}{7} & \multirow[t]{4}{*}{ Lockdown has given me opportunity } & $\begin{array}{l}\text { Spend more time with loved } \\
\text { ones }\end{array}$ & $\begin{array}{l}267 \\
(49.4 \%)\end{array}$ & $\begin{array}{l}350 \\
(46.1 \%)\end{array}$ & $\begin{array}{l}617 \\
(47.5 \%)\end{array}$ & 0.015 \\
\hline & & Binge watch shows & $48(8.9 \%)$ & $99(13 \%)$ & $\begin{array}{l}147 \\
(11.3 \%)\end{array}$ & \\
\hline & & $\begin{array}{l}\text { Spend more time with } \\
\text { myself }\end{array}$ & $\begin{array}{l}144 \\
(26.6 \%)\end{array}$ & $\begin{array}{l}225 \\
(29.6 \%)\end{array}$ & $\begin{array}{l}369 \\
\quad(28.4 \%)\end{array}$ & \\
\hline & & None of the above & $82(15.2 \%)$ & $85(11.2 \%)$ & $\begin{array}{l}167 \\
(12.8 \%)\end{array}$ & \\
\hline \multirow[t]{2}{*}{8} & \multirow[t]{2}{*}{ Lockdown made me learn something new } & No & $82(15.2 \%)$ & $\begin{array}{l}137 \\
\quad(18.1 \%)\end{array}$ & $\begin{array}{l}219 \\
\quad(16.1 \%)\end{array}$ & 0.17 \\
\hline & & Yes & $\begin{array}{l}459 \\
\quad(84.8 \%)\end{array}$ & $\begin{array}{l}622 \\
\quad(81.9 \%)\end{array}$ & $\begin{array}{l}1081 \\
(83.2 \%)\end{array}$ & \\
\hline \multirow[t]{2}{*}{9} & \multirow[t]{2}{*}{ On a personal level I feel, this lockdown has done } & More harm than good & $200(37 \%)$ & $\begin{array}{l}261 \\
(34.4 \%)\end{array}$ & $\begin{array}{l}461 \\
\quad(35.5 \%)\end{array}$ & 0.34 \\
\hline & & More good than harm & $341(63 \%)$ & $\begin{array}{l}498 \\
\quad(65.6 \%)\end{array}$ & $\begin{array}{l}839 \\
(64.5 \%)\end{array}$ & \\
\hline
\end{tabular}


Table 2 continued

\begin{tabular}{|c|c|c|c|c|c|c|}
\hline \multirow[t]{2}{*}{ No. } & \multirow[t]{2}{*}{ Item } & \multirow[t]{2}{*}{ Choices } & \multicolumn{2}{|l|}{ Age } & \multirow[t]{2}{*}{ Total } & \multirow[t]{2}{*}{$p$ value } \\
\hline & & & $<20$ years & $>20$ years & & \\
\hline \multirow[t]{4}{*}{10} & \multirow[t]{4}{*}{ Considering everything that I am going on, I feel } & Safe & $222(41 \%)$ & $232(30.6 \%)$ & $\begin{array}{l}454 \\
(34.9 \%)\end{array}$ & \multirow[t]{4}{*}{0.001} \\
\hline & & Anxious & $81(15 \%)$ & $\begin{array}{l}159 \\
(20.9 \%)\end{array}$ & $\begin{array}{l}240 \\
(18.5 \%)\end{array}$ & \\
\hline & & Scared & $79(14.6 \%)$ & $116(15.3 \%)$ & $195(15 \%)$ & \\
\hline & & Uncertain & $\begin{array}{l}159 \\
(29.4 \%)\end{array}$ & $252(33.2 \%)$ & $\begin{array}{l}411 \\
(31.6 \%)\end{array}$ & \\
\hline \multirow[t]{4}{*}{11} & \multirow[t]{4}{*}{ If I think of going back to normal life, I feel } & Excited & $213(39.4 \%)$ & $\begin{array}{l}290 \\
(38.2 \%)\end{array}$ & $\begin{array}{l}503 \\
(38.7 \%)\end{array}$ & \multirow[t]{4}{*}{0.97} \\
\hline & & Panic & $50(9.2 \%)$ & $72(9.5 \%)$ & $122(9.4 \%)$ & \\
\hline & & Tensed & $173(32 \%)$ & $243(32 \%)$ & $416(32 \%)$ & \\
\hline & & Haven't thought about that & $\begin{array}{l}105 \\
(19.4 \%)\end{array}$ & $154(20.3 \%)$ & $259(19.9 \%)$ & \\
\hline \multirow[t]{3}{*}{12} & \multirow[t]{3}{*}{ Looking back on times before lockdown I feel I was } & Overworked & $\begin{array}{l}158 \\
(29.2 \%)\end{array}$ & $\begin{array}{l}223 \\
(29.4 \%)\end{array}$ & $\begin{array}{l}381 \\
(29.3 \%)\end{array}$ & \multirow[t]{3}{*}{0.95} \\
\hline & & Enjoying routine & $\begin{array}{l}247 \\
(45.7 \%)\end{array}$ & $\begin{array}{l}340 \\
(44.8 \%)\end{array}$ & $\begin{array}{l}587 \\
(45.2 \%)\end{array}$ & \\
\hline & & Not doing enough & $\begin{array}{l}136 \\
(25.1 \%)\end{array}$ & $\begin{array}{l}196 \\
(25.8 \%)\end{array}$ & $\begin{array}{l}332 \\
(25.5 \%)\end{array}$ & \\
\hline \multirow[t]{2}{*}{13} & \multirow[t]{2}{*}{$\begin{array}{l}\text { On getting back to life after lockdown my lifestyle/ } \\
\text { routine would }\end{array}$} & Change & $\begin{array}{l}410 \\
(75.8 \%)\end{array}$ & $\begin{array}{l}614 \\
(80.9 \%)\end{array}$ & $\begin{array}{l}1024 \\
(78.8 \%)\end{array}$ & \multirow[t]{2}{*}{0.026} \\
\hline & & Not change & $\begin{array}{l}131 \\
(24.2 \%)\end{array}$ & $\begin{array}{l}145 \\
(19.1 \%)\end{array}$ & $\begin{array}{l}276 \\
(21.2 \%)\end{array}$ & \\
\hline \multirow[t]{3}{*}{14} & \multirow[t]{3}{*}{ Future world according to me looks } & Bright & $\begin{array}{l}167 \\
(30.9 \%)\end{array}$ & $\begin{array}{l}221 \\
(29.1 \%)\end{array}$ & $\begin{array}{l}388 \\
(29.8 \%)\end{array}$ & \multirow[t]{3}{*}{0.66} \\
\hline & & Bleak & $\begin{array}{l}225 \\
\quad(41.6 \%)\end{array}$ & $\begin{array}{l}313 \\
\quad(41.2 \%)\end{array}$ & $\begin{array}{l}538 \\
\quad(41.4 \%)\end{array}$ & \\
\hline & & Same as before & $\begin{array}{l}149 \\
\quad(27.5 \%)\end{array}$ & $\begin{array}{l}225 \\
(29.6 \%)\end{array}$ & $\begin{array}{l}374 \\
\quad(28.8 \%)\end{array}$ & \\
\hline
\end{tabular}

the two age groups (less than 20 years and more than 20 years) did not vary in most of the situations.

\section{Introspection of the Students}

On introspection on the time spent before the lockdown, $45 \%$ replied that they were enjoying the routine. Twentynine percentage felt overworked, and $26 \%$ felt they were not doing enough. Seventy-nine percentage of the students felt that they need to change their lifestyle and routine postpandemic lockdown. Forty-one percentage of the students felt that the world looked bleak post-lockdown. On the other hand, $30 \%$ felt there was a bright future, while $29 \%$ felt it will be the same as before.

\section{Discussion}

WHO has defined youth as those between 15 and 24 years old. The national survey indicates that $30.9 \%$ of the Indian population are the young people and $32.8 \%$ of this population attend educational institutions [5, 6]. Healthy and productive adulthood with a likelihood of reduced health problems depends largely on the critical youth phase of life where social interactions and relationships undergo constant change. Young people's health is vital and crucial. Youth is usually equated with good health, but as per WHO, an estimated 2.6 million young people aged 10 to 24 years die each year and a much greater number of young people suffer from illnesses 'behaviours' which hinder their ability to grow and develop to their full potential [6]. At least 20 per cent of young people are likely to experience some form of mental illness-such as depression, mood disturbances, substance abuse, suicidal behaviours, eating disorders and others [7-11]. 
Dr. Raghu Krishnamurthy, Consultant Psychiatrist at BGS Gleneagles Global Hospitals, Bengaluru, has observed that the awareness of the correlation of loneliness and depression and anxiety and suicidal tendencies is abysmally low although India is a minefield of mental health concerns. It was also his observation that the paradox of our times that the youth of today prefer isolation while subconsciously seeking company through social media is a major cause for emotional hindrances. The value of genuine interactions is underestimated by all institutions including family [12]. In this study, the young university students, especially the younger group, spent more time with their loved ones during the pandemic. The students from Kerala in this study group were seen to spend more time with their loved ones. This seemed to be a positive impact of the pandemic on the youth of the society.

As the fastest growing economy today, India is home to a fifth of the world's youth. Half of its population of 1.3 billion is below the age of 25 , and a quarter is below the age of 14. India's young population is its most valuable asset and most pressing challenge [2, 7, 11]. It provides India with a unique demographic advantage. But this opportunity will be lost without proportionate investment in human capital development. At the same time, the world today is more dynamic and uncertain than ever before.

With the exponential change in the economic, demographic and technological area in India, the true growth potential of its youth needs to be realized in order to participate adequately and productively in its economy. In this study, it is seen that the young university students are willing to think of alternate ways of engaging oneself. Their sleep and entertainment have not been affected adversely in the initial period of the pandemic, although they have felt unable to concentrate on academic activities due to continuous home stay which reinstate traditional college environment for betterment of studies.

As we are getting to terms with the new normal that has come into being due to this pandemic, we would all come to unthinkingly accept this new normal. Although many are grieving the loss of loved ones and many have lost their jobs, living in quarantine has given many, especially the young, the opportunity to reflect on one's lives and to reset them. It was also revealed that spending more time with themselves was in second place in our study probably due to the influence of gadgets among youngsters. In our study also, the young university students were willing to relax and get refocused in life. The question of the usefulness of the hypercompetitive capitalism seems to have achieved great importance at this time [13].

Quarantine has also allowed many to experiment with new habits and new lifestyles which was seen among the students of this study group. It is understood from this study that the young ones want to put less career pressure as they realize that work is not what matters most in life. Again, it is worth noting that the ability to slow down entails a great deal of privilege which may be the reason that the young university students who do not have to fend for themselves are more relaxed in their attitude towards the situation created by the pandemic.

Most of them were excited about when they think about going back to normal life after lockdown, and the future looked bleak for $41.4 \%$ of students versus $29.8 \%$ who felt bright about their future, implying that though most of the students are temporarily happy due to this unexpected, long time stay in home, they lack confidence in facing their life after lockdown. Appropriate counselling in relation to the above can help to overcome this problem.

When one hits the low in life, one sees who really shows up for you. Many appreciate the family members and friends who have been there for them during tough times. Lockdown seems to have reinforced the necessity of telling people how you feel about them. With social distancing and stay-at-home orders, it is easier to feel isolated from the world. So many have felt that, in order to keep their spirits up, it was important to let people one cares about to know how they feel about them. This quality was seen in our study group, especially among the students from Kerala. Exercising daily was a common response which was done to cope with the lockdown, sleep better, get clearer brain activity, improve one's mood and keep up other good habits.

2.6 billion people, one-third of the world's population, is in some form of lockdown. In late February 2020, right before European countries mandated various forms of lockdown, The Lancet published a review of 24 studies documenting the psychological impact of quarantine [14]. Unsurprisingly, people who were quarantined were likely to develop a wide range of symptoms of psychological stress and disorder. In one study, no less than $28 \%$ of quarantined parents warranted a diagnosis of "trauma related mental health disorder". Among quarantined hospital staff, almost $10 \%$ reported "high depressive symptoms" up to three years after being quarantined [14].

Reasons for stress that abound in lockdown are as follows: risk of infection, fear of becoming sick or of losing loved ones, as well as the prospect of financial hardship [13, 14]. A sharp spike in absenteeism and burnout is expected as per a report [14]. In a benchmark survey before the lockdown in a representative sample of a Belgian population, $32 \%$ of the population could be classified as highly resilient and only $15 \%$ of the population indicated toxic levels of stress. In a later survey after two weeks of lockdown, the highly resilient group shrunk to $25 \%$ of the population and the toxic levels of stress group increased to $25 \%[13,14]$. 
Similarly, in our study, we found that the young university students were relaxed and coping with the lockdown in a positive manner which may be due to the fact that the survey was done in the early days. A further study after longer periods of lockdown may show a more adversely affected youth. This study has therefore brought out the importance of the necessity for support for the young minds as we move forwards tackling the pandemic.

\section{Conclusion}

This study indicates that the young university students who are well provided for by their parents do not seem to be affected adversely by the lockdown due to the pandemic. Of course, this survey was done only a month after the lockdown and the results may change if the survey is done after a longer period of lockdown. So, the suggestions to the government and NGOs from this survey is that measures should be taken to help individuals to cope with the mental health.

The government and NGOs could do the following:

a) Make sure self-help interventions are in place.

b) Educate people about the expected psychological impact and reactions to a lockdown experience.

c) Launch a specific website to address psychosocial issues.

d) Make sure that people with acute issues can find the help they need.

\section{References}

1. Holshue ML, DeBolt C, Lindquist S, Lofy KH, Wiesman J, Bruce H (2020) First Case of 2019 Novel Coronavirus in the United States. N Engl J Med 82:929-936

2. Vannabouathong C, Devji T, Ekhtiari S, Chang Y, Phillips SA, Zhu M, Chagla Z, Main C, Bhandari M (2020) Novel
Coronavirus COVID-19: Current Evidence and Evolving Strategies. J Bone Joint Surg Am 102(9):734-744

3. Branswell H, and Joseph A 2020. WHO declared the coronavirus outbreak a pandemic. Stat News, March 11. https://www.stat news.com/2020/03/11/who-declares-the-coronavirus-outbreak-apandemic/. Accessed May 12, 2020.

4. Peeri NC, Shrestha N, Rahman MS, Zaki R, Tan Z, Bibi S, Baghbanzadeh M, Aghamohammadi N, Zhang W, Haque U (2020) The SARS, MERS and novel coronavirus (COVID-19) epidemics, the newest and biggest global health threats: what lessons have we learned? Int J Epidemiol 49(3):717-726

5. Youth in India. 2017. http://mospi.nic.in/sites/default/files/pub lication_reports/Youth_in_India-2017.pdf. Accessed 1 Nov 2020.

6. Adolescents: health risks and solutions.2018.https://www.who. int/news-room/fact-sheets/detail/adolescents-health-risks-andsolutions. Accessed 1 Nov 2020.

7. Pillai A, Patel V, Cardozo P, Goodman R, Weiss HA, Andrew G (2008) Non-traditional lifestyles and prevalence of mental disorders in adolescents in Goa. India Br J Psychiatry 192:45-51

8. Malhotra S, Kohli A, Kapoor M, Pradhan B (2009) Incidence of childhood psychiatric disorders in India. Indian J Psychiatry 51:101-107

9. Nair MK, Paul MK, John R (2004) Prevalence of depression among adolescents. Indian J Pediatr 71:523-524

10. Sahoo S, Khess CR (2010) Prevalence of depression, anxiety, and stress among young male adults in India: a dimensional and categorical diagnoses-based study. J Nerv Ment Dis 198:901-904

11. Latha KS, Reddy H (2007) Patterns of stress, coping styles and social supports among adolescents. J Indian Assoc Child Adolesc Ment Health 3:5-9

12. Rashmi Vasudeva. https://www.thehindu.com/society/how-lone liness-is-affecting-the-indian-youth/article27115615.ece. Accessed 1 Nov 2020.

13. Brooks SK, Dunn R, Amlôt R, Rubin GJ, Greenberg N (2018) A systematic, thematic review of social and occupational factors associated with psychological outcomes in healthcare employees during an infectious disease outbreak. J Occup Environ Med 60:248-257

14. Brooks SK, Webster RK, Smith LE, Woodland L, Wessely S, Greenberg N, Rubin GJ (2020) The psychological impact of quarantine and how to reduce it: rapid review of the evidence. Lancet 395(10227):912-920

Publisher's Note Springer Nature remains neutral with regard to jurisdictional claims in published maps and institutional affiliations. 